\title{
Il bicchiere a volte è mezzo pieno: buone notizie in arrivo!
}

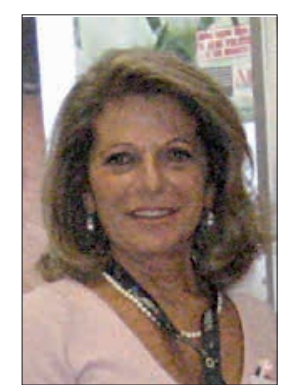

Luisa Sternfeld Pavia

\section{A tutti voi un caloroso abbraccio!}

Voglio condividere con voi le ottime e importantissime notizie che ci riguardano e che rappresentano alcuni degli obiettivi per i quali l'AIRP è sempre in prima linea. Ci sono tante novità di cui parlare e quindi inizierò subito!

Come forse già sapete nei primi giorni dello scorso Gennaio il Primo Ministro Gentiloni ha firmato il DPCM sui nuovi LEA, i Livelli Essenziali di Assistenza, a seguito del via libera delle Regioni. Nel nuovo elenco che dà diritto all'esenzione di alcune prestazioni mediche è stato finalmente inserito anche il Rene Policistico Autosomico Dominante (ADPKD) e, nell'elenco delle Malattie Rare, è stato inserito il Rene Policistico Autosomico Recessivo (ARPKD). La Ministra della Salute Beatrice Lorenzin ha commentato su Twitter che questo è "un passaggio storico per la sanità italiana" e in effetti è davvero un grande passo in avanti perché garantisce i fondamentali diritti ai nostri malati e rende ufficiale il riconoscimento di queste patologie all'interno del sistema della sanità italiana. Lasciatemi dire che siamo davvero soddisfatti di aver realizzato un importante risultato per il quale ci siamo molto impegnati e cogliamo l'occasione per ringraziare sentitamente le Autorità che hanno ascoltato le nostre richieste rispondendo positivamente e con sollecitudine. Siamo certi che questa evoluzione avrà un impatto davvero significativo nella vita di tante persone.

La seconda buona notizia arriva dal Tar del Lazio, che

Accepted: January 30, 2017

Published online: February 15, 2017

Indirizzo per la corrispondenza:

Dr. ssa Luisa Sternfeld Pavia

AIRP Associazione Italiana Rene Policistico onlus

Via A. Bazzini 2

20131 Milano

luisa.sternfeld.airp@renepolicistico.it ha accolto il ricorso dell'Azienda farmaceutica giapponese Otsuka Pharmaceuticals contro l'inserimento in classe C del tolvaptan, che, a tutt'oggi, è l'unica terapia in grado di rallentare la crescita delle cisti renali nel rene policistico (ADPKD) e quindi la progressione della patologia.

AIFA, I'Agenzia Italiana del Farmaco, aveva inizialmente inserito il farmaco in classe $C$ con il conseguente costo

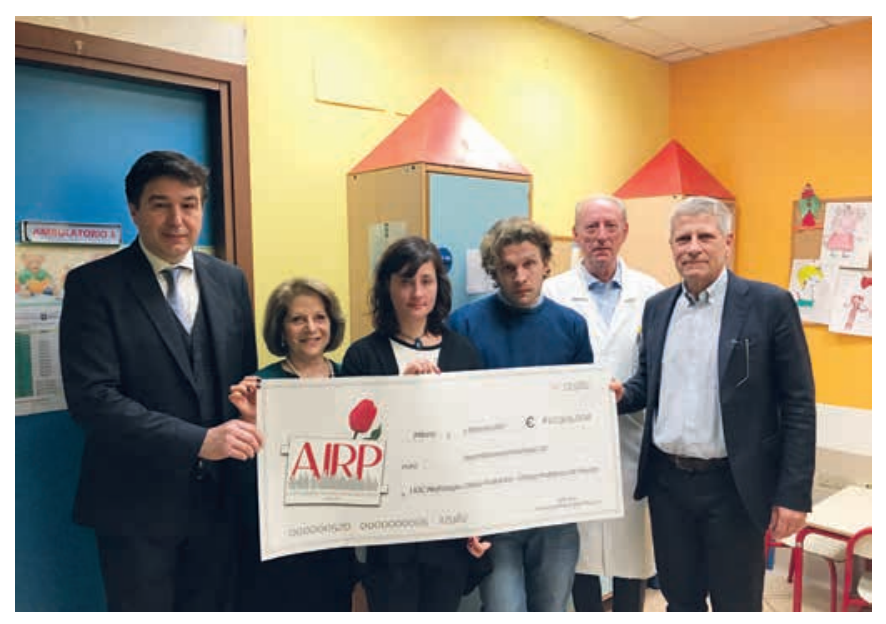

Fig. 1 - La consegna della donazione.

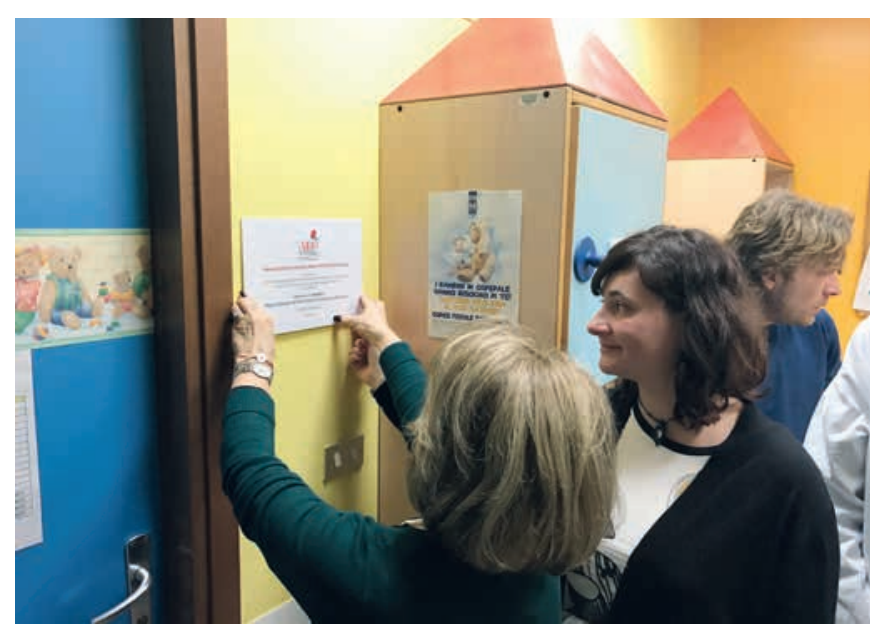

Fig. 2 - La targa. 


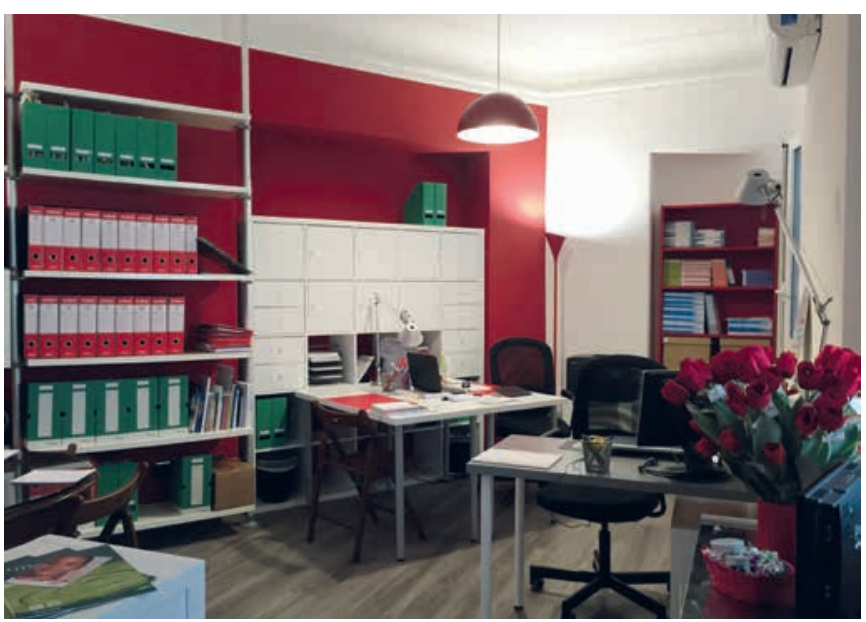

Fig. 3 - La nuova sede AIRP.

annuo a carico dei pazienti. Questo grande passo in avanti riguarda da vicino tutti i 24.000 italiani affetti da rene policistico che, fino a oggi, si sono scontrati con decisioni che negavano loro il diritto all'accesso al tolvaptan. Ora auspichiamo tempi rapidi per l'immissione in commercio del farmaco Jinarc (il cui principio attivo è il tolvaptan), affinché possa essere disponibile nel più breve tempo possibile e in modo equo per tutti i pazienti.

Siamo molto soddisfatti di questa decisione e confidiamo in una collaborazione proficua tra AIFA e l'azienda al fine di rendere il farmaco accessibile in modo rapido ed equo. Come sapete c'è un gap che va colmato: a differenza di quello che succede in altri Paesi, Inghilterra, Germania, Francia, Belgio e Svizzera che da tempo hanno accesso al farmaco, i pazienti italiani sono stati, fino a oggi, costretti ad assistere inermi al peggioramento delle proprie condizioni, arrivando molto spesso a doversi sot- toporre a dialisi o a trapianto già in giovane età. Stiamo monitorando questi sviluppi con grande attenzione e non appena avremo a disposizione ulteriori notizie provvederemo a diramarle con tempestività.

Proseguendo con le novità siamo davvero felici di comunicarvi che il 2 Febbraio è avvenuta la consegna di una donazione liberale (Figg. 1 e 2) per "l'adesione al progetto ARegPKD, Registro Europeo del Rene Policistico Autosomico Recessivo" a favore della Clinica Pediatrica G. e D. De Marchi - Fondazione IRCCS Cà Granda, Ospedale Maggiore Policlinico. II progetto è condotto dal Prof. Giovanni Montini, UOC di Nefrologia e Dialisi Pediatrica. La somma in denaro è stata raccolta dalle vostre donazioni del $5 \times 1000$, dalle donazioni solidali e dalla generosa raccolta fondi promossa dalla mamma e dal papà di Beatrice, \#RicordandoBeatricexdireARPKDstop. In suo ricordo, durante la cerimonia di consegna della donazione, è stata dedicata una targa nell'area giochi presso gli ambulatori di Nefrologia, al primo piano della Clinica Pediatrica De Marchi di Milano.

L'ultimo argomento che vorrei condividere con voi tutti è che l'AIRP ha una nuova sede (Fig. 3), con uffici più spaziosi pergarantire una maggiore capacità organizzativa al grande lavoro che ci attende in futuro. I nostri riferimenti non cambiano perché la nuova sede è allo stesso indirizzo della precedente. Anche il nostro impegno non cambia, sempre al massimo delle nostre possibilità, ma siamo certi che questo positivo sviluppo sarà di ulteriore stimolo a lavorare sempre meglio e con una rinnovata carica di energia.

A chi non l'avesse ancora fatto, ricordo il rinnovo delle quote associative, così importanti per il prosieguo delle nostre attività, e a tutti voi invio un affettuoso saluto!

Luisa Sternfeld Pavia Presidente AIRP 